\title{
Interpretation of NMR diffusometry data regarding droplet size distributions in micro- and nanoemulsions
}

\author{
J.-H. Sommerling ${ }^{a \star}$, A. J. Simon ${ }^{a}$, A. Haber ${ }^{b}$, M. Johns ${ }^{b}$, G. Guthausen ${ }^{c}$, H. Nirschl ${ }^{a}$

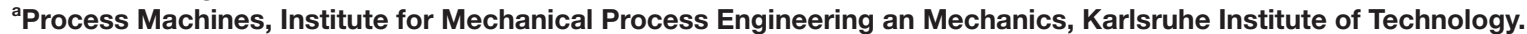 \\ ${ }^{b}$ School of Mechanical and Chemical Engineering, University of Western Australia \\ ${ }^{\mathrm{c}}$ Pro'2NMR, Institute for Mechanical Process Engineering and Mechanics and Institute of Biological Interfaces 4, Karlsruhe Institute of \\ Technology \\ *Corresponding author: jan-hendrik.sommerling@kit.edu
}

\begin{abstract}
Nuclear magnetic resonance (NMR) diffusometry allows to measure droplet size distributions in emulsions. This non-invasive, nondestructive method has several advantages compared to common mainly optical methods for droplet sizing. NMR requires almost no sample preparation, unlike optical methods or cryoTEM, where samples need to be transparent and preparation or dilution is necessary. The established method, combining the concept of Murday and Cotts for diffusion in a limited spherical geometry with Packer and Rees, assuming a log-normal distribution for the droplet sizes, has limitations regarding the distribution and size range of droplets. There are approaches to overcome the limitations raised by the statistical log-normal distribution. A very successful approach is a numerical method, the regularisation, which does not assume any distribution form. In this work, we discuss the interpretation of NMR PFG-STE data leading to droplet size distributions in accordance to the established methods and the regularisation. We also address the problem of the lower size limitations and propose a way to use the Stokes-Einstein equation to gain droplet size distributions (DSD) for emulsions with droplets smaller than $1 \mu \mathrm{m}$ of OW emulsions.
\end{abstract}

\section{Introduction}

mulsions are heavily used in food and pharmaceutical industry, allowing e.g. to enhance flavour, to solubilize hydrophilic in hydrophobic media and vice versa, and to reduce the fat content ${ }^{1,2}$. One of the major characteristics of emulsions, governing its appearance, viscosity, and stability is the droplet size distribution (DSD). It can be challenging to measure DSDs, as all the different techniques have limitations. For example most optical methods require light transmission and therefore need highly diluted samples with continuous phases that cannot be opaque. An established alternative approach for measuring droplet size distributions is NMR diffusometry, which is not limited to low dispersed phase fractions. Based on the geometrically restricted diffusion of the dispersed phase's molecules inside the droplets, the size can be calculated, as described by Murday and Cotts ${ }^{3}$. Additionally, this analytical description of restricted diffusion in a sphere can be combined with a distribution function to account for size distributions of droplets, as done by Packer and Rees ${ }^{4}$, assuming a log-normal distribution. This droplet sizing with NMR pulsed field gradient - stimulated echo sequence (PFG-STE) is well established for simple emulsions with radii larger than about $1 \mu \mathrm{m}$. Even in the case of multiple emulsions, PFG NMR was shown to be applicable revealing the $\mathrm{DSD}$, the dispersed phase ratios as well as exchange between phases ${ }^{5,6}$.

The assumption of a log-normal distribution for the DSD of an emulsion is debatable as it is a statistical but not an experimental distribution. It is usually argued that the droplet size and its distribution is strongly dependent on the production process and formulation, which can lead to an uneven or multimodal distribution for example with a larger fraction of small droplets. To account for this, several approaches have been explored to use other distribution functions. One of the more prominent cases is the numerical approach of regularisation described in literature ${ }^{7-9}$. In this work two approaches for droplet size distributions are compared and discussed with respect to their benefits and restrictions for the interpretation of the diffusion data.

The model developed by Murday and Cotts and expanded by Packer and Rees has limitations for small droplet sizes below about $1 \mu \mathrm{m}$. Droplets in typical food and pharmaceutical emulsions can very well be much smaller than $1 \mu \mathrm{m}$ in practice. Nanoemulsions are especially important for pharmaceutical applications, where sizes below $300-400 \mathrm{~nm}$ are mandatory ${ }^{10}$. The aim is therefore to provide a model for this important class of nanoemulsions to be analysed via NMR. Here, we discuss how to interpret diffusion data from emulsions with droplets smaller than $1 \mu \mathrm{m}$ and offer a possibility to measure the droplet size distribution by modelling the droplet movement with Stokes-Einstein equation.

\section{Material and methods}

Oil in water $(\mathrm{O} / \mathrm{W})$ emulsions with large droplets above $1 \mu \mathrm{m}$ were stabilized with whey protein isolate and xanthan from Sigma Aldrich, increasing the viscosity of the dispersed phase. The dispersed phase fraction was 10\%-vol sunflower oil dispersed in bi-distilled water with a rotor-stator homogenizer Ultraturrax (IKA GmbH) at $10.000 \mathrm{rpm}$. Bimodal emulsions were produced and measured as described in ref. 11.

O/W emulsions with small droplet size below $1 \mu \mathrm{m}$ were produced using 10\%-vol squalene oil ( $\geq 98 \%$ Sigma-Aldrich), bi-distilled water, phospholipid E80 contributed by Lipoid $\mathrm{GmbH}$ as emulsifier from egg source containing 80\% phosphatidylcholine, dispersed with ultrasonication using a Branson 450 sonotrode. 
Droplet sizes were measured at room temperature with a Bruker SWB $200 \mathrm{MHz}$ NMR spectrometer, equipped with a Diff30 probe The PFG-STE pulse sequence was used with a gradient duration $\delta$ of $3 \mathrm{~ms}$, incrementing the gradient amplitude from $1.2 \mathrm{mT} / \mathrm{m}$ to $6 \mathrm{~T} / \mathrm{m}$. In order to confirm the measurements and their interpretation, the diffusion time $\Delta$ was varied from 20 to $400 \mathrm{~ms}$. Droplet size distributions were determined with self-written scripts in Matlab, using either a log-normal distribution following Packer \& Rees or regularisation. Additionally, emulsions with small droplet sizes were measured with dynamic light scattering measurements to confirm the DSD determined by NMR, using a Beckman Coulter N4 plus.

\section{Theory}

The fundamental principle of DSD determination by PFG-NMR is restricted diffusion: Molecules in the dispersed phase of an emulsion are restricted in their translational diffusion by the interface to the quasi-continuous phase. This means that the average mean free path of these molecules is related to the restricting geometry. Depending on the diffusion time and resulting average mean free path, the molecules will experience the boundaries to a different extent. If the diffusion time $\Delta$ is too short, the molecules will not interact with the droplet walls and the restriction will have no impact on the signal decay, while a very long diffusion time will result in an average molecule position in the middle of the limiting droplet geometry, loosing the information about distance to the walls. In between these two extrema, the PFG-NMR signal decay as a function of the gradient amplitude is determined by the size of the restricting geometry, i.e. the droplet's diameter when assuming a spherical geometry ${ }^{3,12}$.

Expanding these expressions by considering the fact that the droplet size in a typical emulsion is not mono-disperse but shows a distribution, Packer and Rees ${ }^{4}$ established a model, assuming a log-normal distribution. Nowadays numerical methods are used to overcome boundary conditions or assumptions of the old models, in the present case the assumption of a log-norm distribution is relaxed by applying regularisation algorithms ${ }^{7,8,13}$.

Apart from the assumptions of spherical geometry and the restriction to suitable time scales for diffusion time and gradient duration, a major limitation of the established method concerns the range of droplet sizes which can be analysed by PFG-NMR. Often, the medians of the DSD's in NMR studies are in the range of $0.5 \mu \mathrm{m}-$ $20 \mu \mathrm{m}$ for $\mathrm{W} / \mathrm{O}$ and $0.25 \mu \mathrm{m}-20 \mu \mathrm{m}$ for $\mathrm{O} / \mathrm{W}^{14}$, which is a severe limitation as a large portion of emulsions are nowadays in the range of a few hundrednm. A different approach can be investigated in this case: When considering the droplets as particles, their thermal translational diffusion can be described by the Stokes-Einstein equation $D=\left(k_{B} T\right) /(6 \pi \times \eta \times r)$, where $D$ is the diffusion coefficient, $k_{B}$ the Boltzmann constant, $T$ the absolute temperature, $\eta$ the viscosity and $r$ the hydrodynamic radius of the particle. Assuming that the molecules inside the droplets experienced the droplet walls frequently, the average position is essentially the centre of the droplet. In this case the inner-droplet diffusion can be neglected, and the thermal motion is determined by the diffusion of the droplet itself. Combining therefore the Stokes-Einstein equation with the approach of Packer and Rees, an alternative way of DSD determination by PFG-NMR can be realised for nanoemulsions. Strictly speaking the Stokes Einstein equation is only valid for diluted systems. However, modifications are known which incorporate the concentration of the dispersed phase ${ }^{15}$. For fairly concentrated nano-emulsions, this approach might be a possibility to determine DSD by PFG-NMR with its known advantages.

\section{Results \\ Comparing the established model with regularisation}

Classical droplet sizing according to Murday and Cotts $^{3}$ is often implemented with a log-normal distribution function as established by Packer \& Rees ${ }^{4}$. This distribution function might not always be suited for emulsion systems, where the size and distribution are known to depend on production parameters and formulation. Therefore both approaches, the analytical log-normal and the numerical regularisation were applied on mono- and bimodally distributed emulsions.

In a first step a mono-modal emulsion with droplet sizes in the range of several micrometres was produced. Both approaches, the established method and the numerical regularisation described successfully the signal decay leading to very similar DSDs (Figure 1) with a median in the range of 1 to $1.5 \mu \mathrm{m}$ and a rather monodisperse distribution, the numerical DSD exhibiting a slightly larger distribution width. The assumption of a lognormal distribution leads to a smoother curve, due to its analytical character, while the regularisation uses discrete intervals which are then displayed and are not as smooth. In the case of irregular distribution this flexibility and variability of the numerical approach is very beneficial. In the present case of food related emulsions with smooth DSD's, the findings cannot be interpreted as advantage for any of the methods. Both methods could be applied and compared with respect to the residuals to estimate their validity respectively. Therefore more demanding distributions were analysed.

NMR is thought to be not capable to reveal multimodality of droplet size distributions. This predominantly is, however, a question of software implementation rather than a question of measurement and restrictions on principle. An emulsion known to show a bimodal DSD was analysed ${ }^{11}$. Figure 2 shows the logarithmic signal decay $\log \left(S / S_{0}\right)$ over $q^{2}=\gamma^{2} \cdot g^{2} \cdot \delta^{2}$ of this emulsion as a function of diffusion time, with the usual monomodal fit (Figure 2a), and a two component fit (Figure 2b), according to Murday-Cotts with a monoand a bimodal lognormal distribution. As displayed in Figure 2a) the signal decay cannot be modelled sufficiently well with a monomodal $\mathrm{DSD}$, leading to large residuals and obvious deviations between

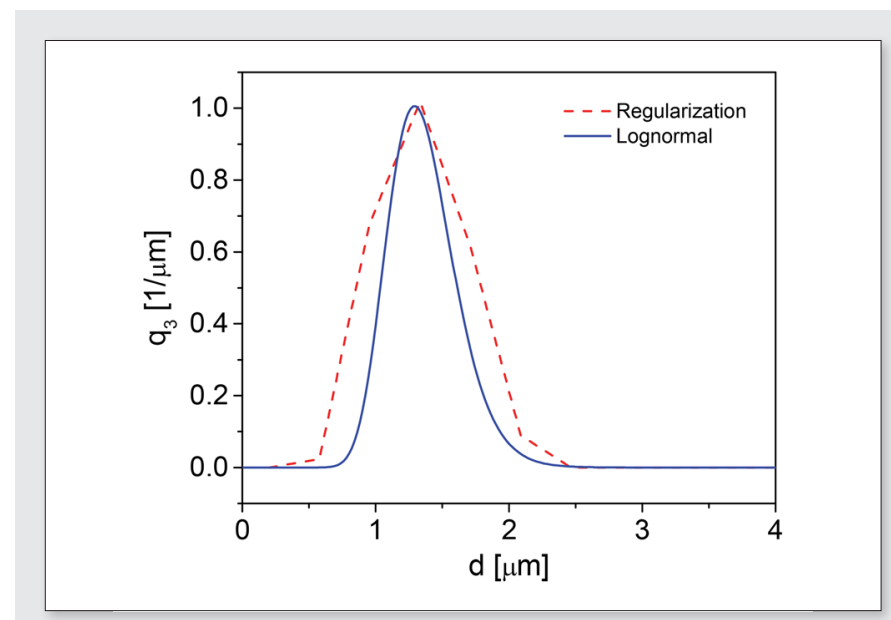

Figure 1. Droplet size distribution of an oil in water emulsion: Both approaches for data interpretation lead to similar DSD. 


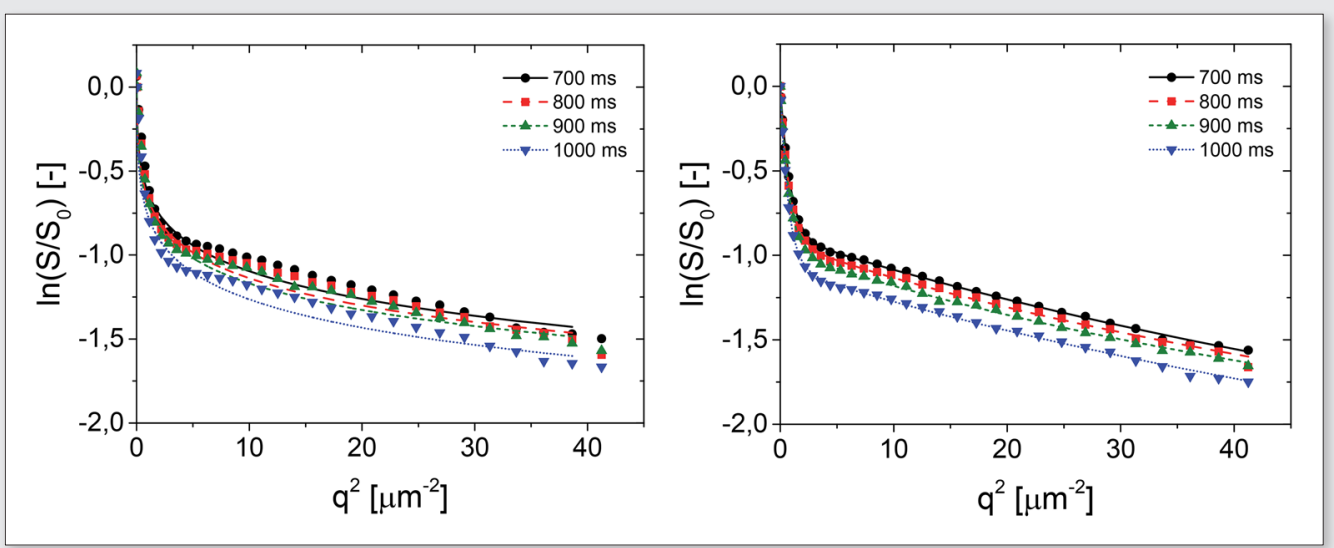

Figure 2. Signal decay of a bimodal DSDs modelled with a) a monomodal and b) a bimodal log-normal distribution according to Murday and Cotts, leading to a considerably better fit with smaller residuals in b).

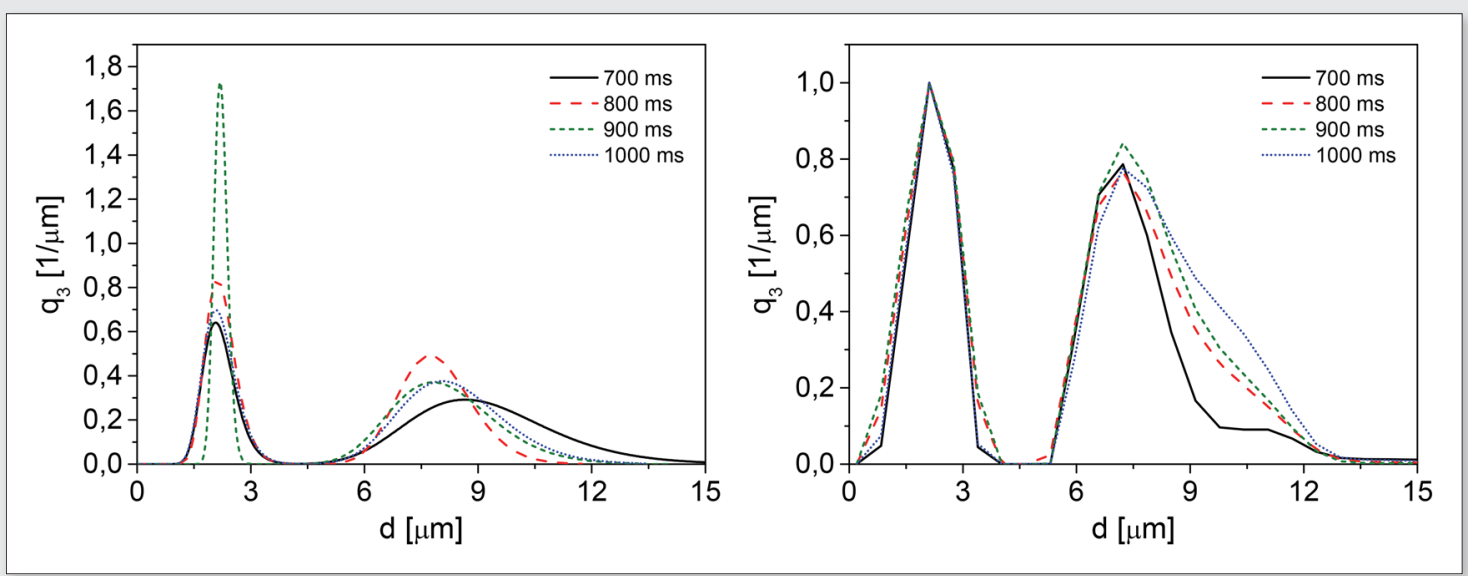

Figure 3. DSD calculated from the signal decay fit in Figure 2b). The bimodal log-normal model a) and the regularisation b) lead to similar DSD, revealing two fractions with a median at $2.5 \mu \mathrm{m}$ and a second median around $7.5 \mu \mathrm{m}$.

measurement and model. A DSD can be calculated, but will be incorrect due to the model error. This DSD can also be viewed as a misinterpretation of the experimental data. The bimodal model of DSD (Figure 2b) describes the signal decay quite well, resulting in very small residuals. The choice of a bimodal log-normal distribution can be concluded to be a good approximation and had as output the bimodal distribution (Figure 3a). The application of the numerical regularisation also leads to an equivalently good fit, identical to Figure $2 b$ ), resulting in a very similar DSD, shown in Figure $3 b$. When compared, both methods display the bimodality of the emulsion and only show slight differences in the fractions of larger droplets. In both cases the goodness of fit has to be considered. While the lognormal distribution is more prone to underestimate the DSD with a simplified model and a priori assumptions, the regularisation can also overfit the data, due to its numerical character. If the algorithm is performed with too many iterations, this will lead to an interpretation of noise, giving more polydisperse distributions.

\section{Nano emulsions analysed by PFG-NMR}

Using squalene in water and E80 as emulsifier, a stable emulsion with very small droplet sizes and a narrow distribution was produced. The droplet size was measured with dynamic light scattering in a high dilution, resulting in a volume weighted median of DSD of approximately $300 \mathrm{~nm}$. In Figure 4 the signal decay $S / S_{0}$ shows a strong dependence on the diffusion time $\Delta$, which is to be expected as already described in Stejskal-Tanner equation ${ }^{16}$. In the case of emulsions, the relative signal decay can be described by a combination of a distribution function $P(r)$ of the radius $r$, which in case of the Murday-Cotts model describes the decay in a limited geometry. $r_{50,3}$ and $\sigma$ are the volume weighted median droplets radius and the width of the distribution, respectively. The signal attenuation $E$ is then given by the following formula:

$$
\frac{S\left(\delta, \Delta, g, D, r_{50,3}, \sigma\right)}{S_{0}\left(\delta, \Delta, 0, D, r_{50,3}, \sigma\right)}=\frac{\int_{0}^{\infty} r^{3} \cdot P(r) \cdot E(\delta, g, \Delta, D, r) d r}{\int_{0}^{\infty} r^{3} P(r) d r}
$$

Also the model of Murday and Cotts itself depends on $\Delta$. Measuring the signal attenuation as a function of $\Delta$ allows an intrinsic validation of the data interpretation as the DSD, unlike the signal attenuation, should not depend on $\Delta$ in single emulsions. Additionally, measurement errors can be detected this way. Namely, a well described problem in NMR measurements is convection in the sample, often due to temperature gradients or inhomogeneity in the sample. Convectional flow will contribute to the signal decay in PFG 


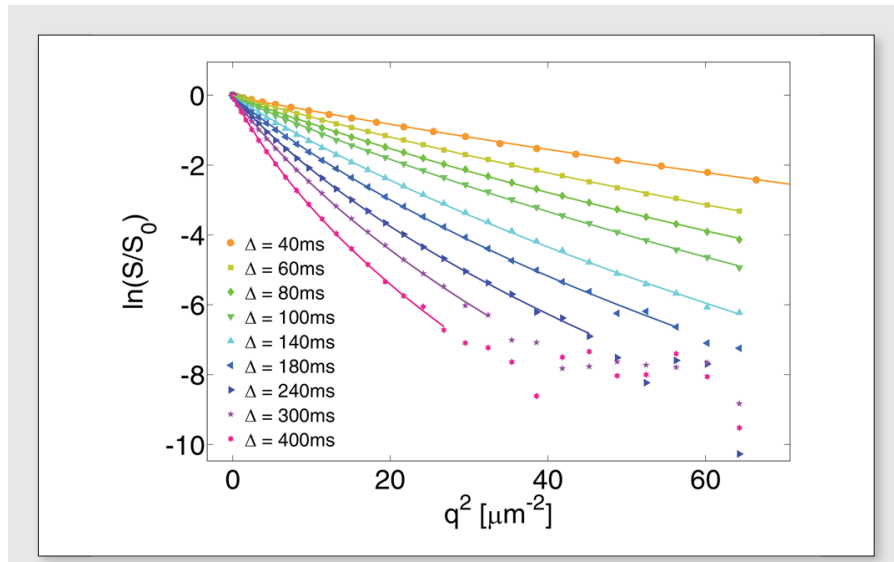

Figure 4. PFG-STE signal decay measured as a function of the diffusion time $\Delta$ of a nano-emulsion.

experiments, changing the resulting diffusion value to a facilitated diffusion. In the case of very small droplets in emulsions, the movement of droplets starts contributing to the signal decay, leading to a different approach for the signal attenuation in a PFG-STE experiment on nanoemulsions:

Case: Stokes-Einstein droplets diffusion:

$$
E(\delta, g, \Delta, D, r)=\exp \left(-\gamma^{2} g^{2} \delta^{2} \cdot\left(\Delta-\frac{\delta}{3}\right) \cdot \frac{k_{b} T}{6 \pi \eta r}\right)
$$

The movement of small droplets or molecules due to the thermal energy is described by the Stokes-Einstein equation. This movement will be more prominent in an oil in water emulsion, because the mobility is proportional to the reciprocal viscosity of the continuous phase, where water with a considerably small viscosity leads to a high mobility of a droplet. The estimated droplet displacement of a $300 \mathrm{~nm}$ oil droplet in water is several times its size, making the Stokes-Einstein movement the larger impactor on the measured displacement in an NMR experiment. The equation could be expanded by consideration of obstruction effects in case of higher concentrations of the dispersed phase.

The deviating signal decays in Figure 4 lead to quite divergent DSDs, if interpreted with a Murday-Cotts restricted model
(Figure 5a). This can be misleading, because the signal decay is not solely determined by the walls when the droplet is moving in the observed time frame. As described before, with long diffusion times $\Delta$, the molecular displacement within the droplet is averaged to its geometrical centre. More correctly, the displacement can be modelled with the Stokes-Einstein equation, which can also be combined with a distribution function, like discussed above for the Murday-Cotts approach. Figure 5b) shows the resulting DSD of a Stokes-Einstein fit with a log-normal distribution for $P(r)$, allowing a good fit of the measurement data and a consistent, e.g. a $\Delta$ independent DSD. The DSD for all diffusion times $\Delta$ is converging to a single median size at $300 \mathrm{~nm}$ which was also determined with dynamic light scattering. We therefore conclude this as a valid approach to estimate droplet sizes below $1 \mu \mathrm{m}$, where the droplet movement is the main contributor to the signal decay.

\section{Conclusion}

We successfully showed for a mono- and a bimodal emulsion that the DSD could be modelled with log-normal and regularisation distribution functions, following the Murday-Cotts approach. Both distributions gave equivalent results, if the goodness of fit to the experimental data is sufficient. In case of more diverse distributions the regularisation is more suitable to describe the measurement data.

Small emulsions with DSD below $1 \mu \mathrm{m}$ are governed by droplet movement according to Stokes-Einstein equation. The signal decay of such emulsions is strongly dependent on the diffusion time, to an extent that cannot be covered by the Murday-Cotts model for limited diffusion within rigid droplets. More so, Stokes-Einstein in combination with a distribution function can be used to translate the gained diffusion distribution into a size distribution. In a next step, the concentration dependence of the Stokes-Einstein interpretation will be implemented allowing the DSD determination of concentrated nano-emulsions.

\section{References}

1. S.E. Friberg, K. Larsson and J. Sjoblom, Food Emulsions. Marcel Dekker Inc., (2004).

2. T.G. Mason, J.N. Wilking, K. Meleson, C.B. Chang and S.M. Graves, "Nanoemulsions: Formation, Structure, and Physical Properties", J.
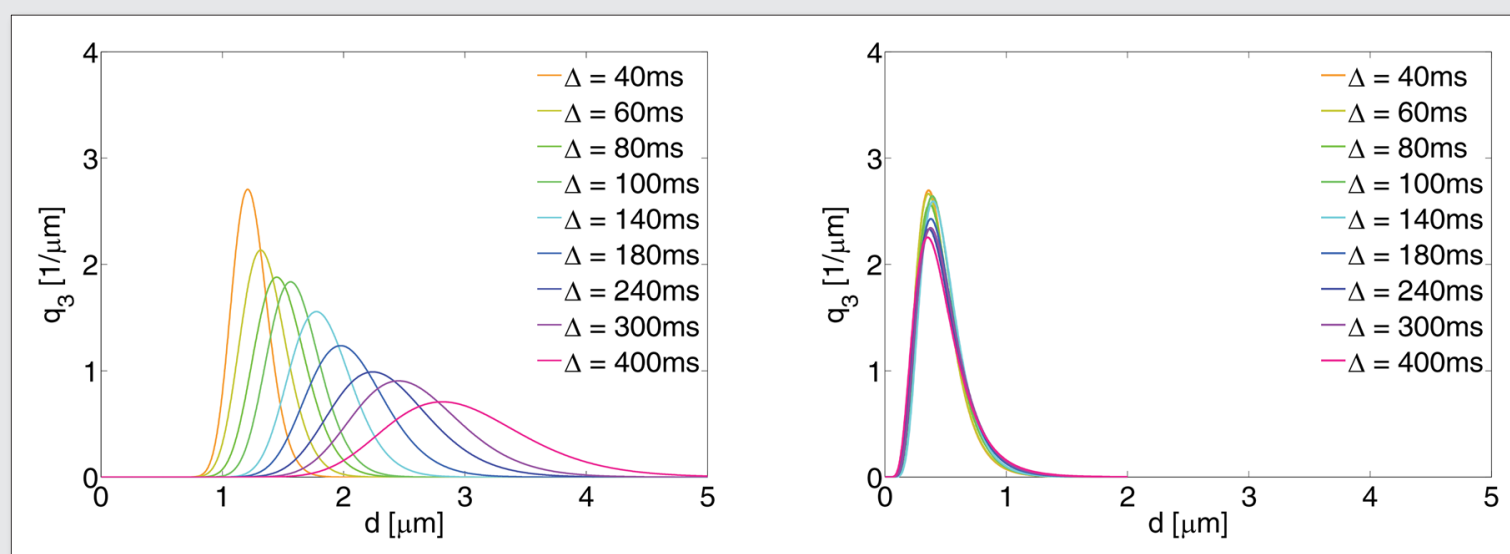

Figure 5. Log-normal DSDs calculated from a fit to the signal decay in Figure 4, with a) a Murday-Cotts approach leading to a physically impossible $\Delta$ dependent distribution and b) a Stokes-Einstein model with a consistent DSD. 
Phys.: Condens. Matter 635 (2006). doi: http://dx.doi.org/10.1088/09538984/18/41/R01

3. J.S. Murday and R.M. Cotts, "Self-Diffusion Coeffcient of Liquid Lithium", J. Chem. Phys. 48, 4938 (1968). doi: http://dx.doi. org/10.1063/1.1668160

4. K.J. Packer and C. Rees, "Pulsed NMR Studies of Restricted Diffusion. 1. Droplet Size Distributions in Emulsions", J. Colloid Interf. Sci. 40, 206 (1972). doi: http://dx.doi.org/10.1016/0021-9797(72)90010-0

5. R. Bernewitz, U.S. Schmidt, H.P. Schuchmann and G. Guthausen, "Structure of and Diffusion in O/W/O Double Emulsions by CLSM and NMR - Comparison with W/O/W", J. Colloids and Surfaces A: Physicochemical and Engineering Aspects 458, 10 (2014). doi: http://dx.doi. org/http://dx.doi.org/10.1016/j.colsurfa.2014.01.002

6. L. Vermeir, P. Sabatino, M. Balcaen, A. Declerck, K. Dewettinck, J.C. Martins, G. Guthausen and P. Van der Meeren, "Effect of Molecular Exchange on Water Droplet Size Analysis as Determined by Diffusion NMR: The W/O/W Double Emulsion Case", J. Colloid Interf. Sci. 475, 57 (2016). doi: http://dx.doi.org/http://dx.doi.org/10.1016/j. jcis.2016.04.029

7. I.A. Lingwood, T.C. Chandrasekera, J. Kolz, E.O. Fridjonsson and M.L. Johns, "Emulsion Droplet Sizing Using Low-Field NMR with Chemical Shift Resolution and the Block Gradient Pulse Method", J. Magn. Reson. 214, 281 (2012). doi: http://dx.doi.org/10.1016/j.jmr.2011.11.020

8. M.L. Johns, "NMR Studies of Emulsions", Curr. Opin. Colloid In. 14, 178 (2009). doi: http://dx.doi.org/10.1016/j.cocis.2008.10.005

9. K.G. Hollingsworth and M.L. Johns, "Measurement of Emulsion Droplet Sizes Using PFG NMR and Regularization Methods", J. Colloid Interf. Sci. 258, 383 (2003). doi: http://dx.doi.org/10.1016/S0021$\underline{9797(02) 00131-5}$
10. K. Westesen and T. Wehler, "Investigation of the Particle Size Distribution of a Model Intravenous Emulsion", J. Pharm. Sci.-Us. 82, 1237 (1993). doi: http://dx.doi.org/http://dx.doi.org/10.1002/jps.2600821211

11. R. Bernewitz, G. Guthausen and H.P. Schuchmann, "NMR on Emulsions: Characterisation of Liquid Dispersed Systems", Magn. Res. Chem. 49, S93 (2011). doi: http://dx.doi.org/10.1002/mrc.2825

12. W.S. Veeman, "Diffusion in a Closed Sphere", Annu. Rep. NMR Spectr. 50, 201 (2003). doi: http://dx.doi.org/10.1016 S0066-4103(03)50005-9

13. L. Ambrosone, S. Murgia, G. Cinelli, M. Monduzzi and A. Ceglie, "Size Polydispersity Determination in Emulsion Systems by Free Diffusion Measurements Via PFG-NMR", The Journal of Physical Chemistry 108, 18472 (2004). doi: http://dx.doi.org/10.1021/jp046480o

14. M.L. Johns and K.G. Hollingsworth, "Characterisation of Emulsion Systems Using NMR and MRI”, Prog. Nucl. Magn. Reson. Spectr. 50, 51 (2007). doi: http://dx.doi.org/10.1016/j.pnmrs.2006.11.001

15. T. Garasanin, T. Cosgrove, L. Marteaux, A. Kretschmer, A. Goodwin and K. Zick, "NMR Self-Diffusion Studies on PDMS Oil-in-Water Emulsion", Langmuir 18, 10298 (2002). doi: http://dx.doi.org/10.1021/la026109x

16. G.J.W. Goudappel, J.P.M. van Duynhoven and M.M.W. Mooren, "Measurement of Oil Droplet Size Distributions in Food Oil Water Emulsions by Time Domain Pulsed Field Gradient NMR", J. Colloid Interf. Sci. 239, 535 (2001). doi: http://dx.doi.org/10.1006/jcis.2001.7603

17. D.W. de Kort, J.P.M. van Duynhoven, H. Van As and F. Mariette, "Nanoparticle Diffusometry for Quantitative Assessment of Submicron Structure in Food Biopolymer Networks", Trends Food Sci. Technol. 42, 13 (2015). doi: http://dx.doi.org/10.1016/.t.tifs.2014.11.003

18. E.O. Stejskal and J.E. Tanner, "Spin Diffusion Measurements: Spin Echoes in the Presence of a Time-Dependent Field Gradient”, J. Chem. Phys. 42, 288 (1965). doi: http://dx.doi.org/10.1063/1.1695690 